\title{
The Knowledge Cartography - A new approach to reasoning over Description Logics ontologies
}

\author{
Krzysztof Goczyła, Teresa Grabowska, Wojciech Waloszek, \\ Michał Zawadzki \\ Gdańsk University of Technology, Department of Software Engineering, \\ ul. Gabriela Narutowicza 11/12, 80-952 Gdańsk, Polska \\ \{kris, tegra, wowal, michawa\}@eti.pg.gda.pl
}

\begin{abstract}
The paper presents a new method for representation and processing ontological knowledge - Knowledge Cartography. This method allows for inferring implicit knowledge from both: terminological part (TBox) and assertional part (ABox) of a Description Logic ontology. The paper describes basics of the method and gives some theoretical background of the method. Knowledge Cartography stores and processes ontologies in terms of binary signatures, which gives efficient way of querying ontologies containing numerous individuals. Knowledge Cartography has been applied in KASEA - a knowledge management system that is being developed in course of a European integrated research project called PIPS. Results of efficiency experiments and ideas of further development of the system are presented and discussed.
\end{abstract}

\section{Introduction}

Rapid development of Internet reveals new needs for exploring and processing data that are stored in resources of the global Web. There is a steady interest among researches (particularly - knowledge engineers) in providing theoretical backgrounds and building practically applicable prototypes of systems that would be able to efficiently integrate, use and exploit Web resources. These systems should be able to assimilate the newly acquired data with the data that already have been acquired and stored in data and knowledge bases. It is clear that data coming from different sources cover only partially a given area of interest, so it is of utmost importance that a powerful data acquisition system should be equipped with a reasoning engine that could infer new facts, not explicitly stated in the data.

To this aim, a knowledge representation method is necessary. In this paper, we present a novel method of knowledge representation called Knowledge Cartography. This method is based on Description Logic (DL) [1] - a decidable part of first-order logic that is aimed at describing terminology systems. Description Logic (actually, one of DL dialects, because DL is in fact a family of formalisms of different levels of expressiveness) became a 
2 Krzysztof Goczyła, Teresa Grabowska, Wojciech Waloszek, Michal Zawadzki

basis of OWL (Web Ontology Language, [2]) that is a standard recommended by W3C Consortium for development of Web ontologies. In this context, the work presented in this paper, contributes to the Semantic Web initiative [3] that strives for development of standard technologies for knowledge management over the Web, particularly for making Web sources semantics machine-readable.

The Knowledge Cartography, primarily proposed by one of the authors of this paper, W. Waloszek, has been applied in a prototype knowledge management system called KASEA (Knowledge Signatures Analyzer). KASEA is a part of a system PIPS (Personal Information Platform for Life and Health Services) [4] that is being developed within a $6^{\text {th }}$ European Union Framework Programme integrated project (priority "e-Health") involving 17 partners from 12 countries, mainly from EU. PIPS's main goal is to create a Web infrastructure to support health and promote healthy life style among European communities. PIPS concentrates on providing health-related assistance to its users: citizens, patients and healthcare professionals. PIPS serves citizens and patients by helping them in making decisions concerning their everyday practices and deciding whether they should consult their doctors. PIPS can also be used by healthcare professionals and can provide them with healthrelated knowledge and advise about course of therapies.

PIPS system must efficiently process large (particularly - in terms of number of individuals) ontologies. The KASEA system allows both for inferring and for storing the inferred results (in this way it somehow resembles the InstanceStore system [5] that also stores results of its inferences but in quite different way). The price we pay for this is a slight reduction of expressiveness of queries that can be submitted to the system and longer time of ontology loading. These limitations, however, turned out not to be critical for the system clients.

The rest of the paper is organized as follows. Section 2 describes the basics of Knowledge Cartography. Section 3 illustrates implementation issues of KASEA. Section 4 presents results of performance analysis and tests in comparison with other DL reasoners. Section 5 summarizes the paper by discussing limitations of the presented approach and proposing the ways of its further development.

\section{The Knowledge Cartography}

Motivation behind the Knowledge Cartography is based on the three assumptions:

1. Terminological part of the knowledge base (TBox) is updated so rarely that it might be considered constant in time. 
The Knowledge Cartography - A new approach to reasoning over Description Logics ontologies 3

2. A knowledge base is queried much more often than updated (by updating we understand addition of new ABox assertions). Therefore performance of information retrieval is crucial, while performance of updating is less critical.

3. A knowledge base should be able to hold and efficiently process information about large numbers of individuals.

On the basis of these assumptions a cartographic approach has been developed. It aims at storing in the knowledge base as many conclusions about concepts and individuals as possible. The conclusions can be quickly retrieved in the process of query answering and remain valid due to the fact that terminology cannot be updated. By proper organisation of the knowledge base (see Sec. 3.1) the same conclusions can be applied to any number of individuals, facilitating information retrieval and reducing size of the base.

\subsection{A General Idea}

The Knowledge Cartography takes its name after a map of concepts. A map of concepts is basically a description of interrelationships between concepts in a terminology. The map is created in the course of knowledge base creation. A map of concepts can be graphically represented in a form similar to a Venn diagram (Fig. 1). Each atomic region (i.e. a region that does not contain any other region) represents a unique valid intersection of base concepts. By valid we mean an intersection that is satisfiable with respect to a given terminology. Intersections of concepts that are not allowed by terminological axioms are excluded from the map (as in Fig. 1b, where two additional axioms eliminated four regions from the map). Cartographer calculates a number of valid atomic regions $n$ and assigns each atomic region a subsequent integer number from the range $[1, n]$. Because any region in the map consists of some number of atomic regions it can be represented by an array of binary digits of length $n$ with " 1 "s in positions mapped to contained atomic regions and " 0 "s elsewhere.

Using this technique we can assign any concept in the terminology a signature - an array of binary digits representing a region covered by the concept in the map. In this way we can describe any combination of complement, union and intersection of described concepts by simply mapping these operations to Boolean negation, disjunction and conjunction.

Formally, we define a function $s$ from concepts to elements of a Boolean algebra $\mathbb{B}^{n}=\{0,1\}^{n}$. The only condition that should be met by the function $s$ to have all desired characteristics is:

$$
s(C) \leq s(D) \Leftrightarrow C \sqsubseteq D
$$




\section{Krzysztof Goczyła, Teresa Grabowska, Wojciech Waloszek, Michal Zawadzki}

In any such function atomic regions are mapped to atoms of the algebra, i.e. arrays of " 0 "s with a sole "1". Moreover, for any concepts $C$ and $D$ the following equalities hold:

$$
s(\neg C)=\neg s(C), s(C \sqcap D)=s(C) \wedge s(D), s(C \sqcup D)=s(C) \vee s(D)
$$

After determination of $s$ function one can solve any TBox reasoning problem (after reducing it to subsumption [1] on the basis of (1)) by signature calculations. Namely:

- query about equivalence of concepts $C$ and $D$ can be performed by checking whether $s(C)=s(D)$,

- query about subsumption of concepts $C$ and $D$ can be performed by checking whether $s(C) \leq s(D)$,

- query about disjointness of concepts $C$ and $D$ can be performed by checking whether $s(C) \wedge s(D)=\{0\}^{n}$.

It can be proven that for any $\mathcal{A L C}$ terminology $\mathcal{T}$ that does not contain $\exists R$.C and $\forall$ R.C constructors we can create the function $s$ for which (1) and (2) hold.

Following the (1) we can notice that the order of the range of $s$ should be equal to the number of terminologically unequivalent concepts that can be expressed. With introduction of $\exists R$.C and $\forall R$.C constructors the latter number can easily reach infinity (namely $\aleph_{0}$ ) rendering direct use of signatures infeasible.

a)

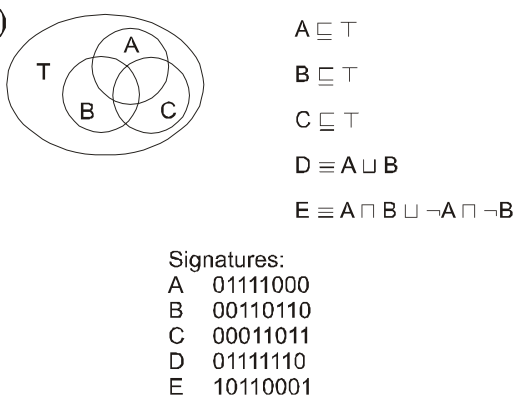

b)

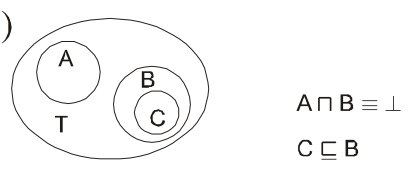

Fig. 1. A map of concepts (a) with two terminological axioms added (b).

Because of this fact, we have made an important decision restricting the use of $\exists R$.C and $\forall R$.C constructors in queries. The only concepts of the form $\exists R$.C and $\forall R$.C that can be used in queries are those explicitly included in the ontology. In that way we restrict the number of expressible terminologically unequivalent concepts. This restriction limits capabilities of the system, but 
The Knowledge Cartography - A new approach to reasoning over Description Logics ontologies 5

our experiences show that this limitation is not critical for knowledge base users.

The same techniques as for TBoxes can be applied to ABox reasoning. We can assign each individual $a$ in ABox a signature of the most specific concept (we denote this concept $C_{a}$; this concept need not to be defined explicitly in the terminology).

After determination of signatures for individuals we can reduce ABox reasoning problems to TBox reasoning problems which in turn can be solved by signature calculations. For example, an instance checking problem (check if an individual $a$ is a member of a concept $C$ ) can be reduced to a question whether the concept $C_{a}$ is subsumed by the concept $C$.

\subsection{The Map Creation Algorithm}

The key algorithmic problem in Knowledge Cartography is determination of function $s$, i.e. map of concepts. We define this problem as follows: for input $\mathcal{A L C}$ terminology $\mathcal{T}$ for each atomic concept and each concept of the form $\exists R$.C ( $\forall$ R.C are converted to the equivalent form $\neg \exists R . \neg C$ ), called together mapped concepts, generate the sequence of bits describing its signature.

The problem itself cannot be polynomial, unless $\mathrm{P}=\mathrm{NP}$, because of its reducibility to CNF-satisfiability. However use of some optimization techniques can make this process efficient for real-life ontologies.

For creation of signatures we base on the fact that atomic regions are mapped to the atoms of Boolean algebra being a range of the function $s$. Atomic regions can be viewed simply as valid intersections of all mapped concepts or their complements in the terminology, i.e. all possible complex concepts of the form:

$$
L_{1} \sqcap L_{2} \sqcap \ldots \sqcap L_{n}
$$

where $n$ is the number of the mapped concepts and $L_{i}$ is a placeholder for $i$-th mapped concept or its complement.

Using this approach we may view a terminology as a set of first-order logic formulae, mapped concepts as variables, and reduce the problem to finding a truth table for the terminology. Each satisfiable combination of variable values may be treated as an atomic region.

From among many techniques available we decided to exploit Ordered Binary Decision Diagrams (henceforth OBDD) originally proposed by Bryant in [7]. In our approach we systematically build a tree of the whole terminology by combining it with logical "AND" operation with the tree generated for formulae corresponding to consequent axioms. Axioms are converted into first order logic formulae in accordance with [7] but the method is somehow simplified because of the fact that concepts of the form 
6 Krzysztof Goczyła, Teresa Grabowska, Wojciech Waloszek, Michal Zawadzki

$\exists R . C$ and $\forall R$.C are represented as a single variable. Each new mapped concept is given a new variable name.

For example, the axiom:

$$
\text { Momo } \equiv \text { Person } \sqcap \forall \text { hasChild.Man } \sqcap \exists \text { hasChild.Man }
$$

would be converted to the form:

$$
\text { Momo } \equiv \text { Person } \sqcap \neg \exists \text { hasChild. } \neg \text { Man } \sqcap \exists \text { hasChild.Man }
$$

and consequently to the formula:

$$
c_{1} \leftrightarrow c_{2} \wedge \neg e_{1} \wedge e_{2}
$$

The outline of the main part algorithm is presented below:
Input: A terminology $\mathcal{T}$ in $\mathcal{A L C}$
Output: An OBDD $T$ for terminology $\mathcal{T}$
1. Initiate $T$ to OBDD of any true formula.
2. For each axiom $A$ from $\mathcal{T}$ :
3. Convert $A$ to the formula $F$ in the way described above.
4. Generate the OBDD $U$ for the formula $F$.
5. $T:=T \wedge U($ where $\wedge$ denotes conjunction of two OBDD trees [7])
6. Next

Direct application of the algorithm to specific terminologies may lead to generation of spurious atomic regions, as shown in Fig. 2a. According to the terminology an individual cannot belong to $\exists R . B$ not belonging to $\exists R . A$ (because each member of $B$ is a member of $A$ ). In order to avoid this effect we perform post-processing.

The post-processing produces a tree $T^{\prime}$ on the basis of $T$. The postprocessing may be basically brought down to checking whether for each combination of values of variables $e_{i}$ that concern the role $R$ and are satisfiable in accordance with $T$ it is possible to create a set of individuals that would satisfy the combination. In the example from Figure 2a the following atomic intersection is satisfiable in accordance to $T$ :

$$
\neg A \sqcap \neg B \sqcap C \sqcap \neg \exists R . A \sqcap \exists R . B
$$

But this region is excluded from $T^{\prime}$ during post-processing because the following family of concepts:

$$
\neg A \sqcap B \sqcap \ldots
$$


The Knowledge Cartography - A new approach to reasoning over Description Logics ontologies 7

is not satisfiable in accordance with $T$ (there can not exist any individual that belongs to $B$ and does not belong to $A$ ).

a)

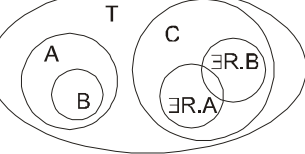

b)

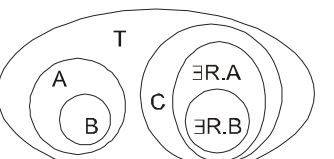

$A \sqcap C \equiv \perp$

$B \sqsubseteq A$

$\exists R . A \sqsubseteq C$

$\exists R . B \sqsubseteq C$

Fig. 2. A map of concepts (a) with two terminological axioms added (b).

The outline of the post-processing algorithm is presented below:

Input: An OBDD $T$ produced in the course of processing Output: An OBDD $T^{\prime}$ with further regions excluded

1. Initiate $T$ ' to $T$.

2. Repeat

3. For each role $R$ from $\mathcal{T}$ :

4. For each combination of $e_{i}$ satisfiable in accordance with $T^{\prime}$ :

5. Check whether it is possible to create a set of individuals that being role $R$ fillers of some individual $a$ would make $a$ satisfy the intersection of concepts of the form $\exists R$.C or $\neg \exists R$.C implied by values of $e_{i}$.

$6 . \quad$ If there is no such set then:

7. Create the formula $F$ making the combination of values unsatisfiable.

8. Generate an OBDD $U$ for the formula $F$.

9. $T^{\prime}:=T^{\prime} \wedge U$

10. End if

11. Next

12. Next

13. Until no changes to $T^{\prime}$ ' have been made.

It can be shown that the post-processing is sound and complete, i.e. all intersections it excludes are invalid and there is no invalid intersection it does not exclude. 
8 Krzysztof Goczyła, Teresa Grabowska, Wojciech Waloszek, Michal Zawadzki

\section{The KaSEa System}

The Cartographic Approach has been successfully exploited in the PIPS system. The Knowledge Inference Engine (KIE), a vital component of the KaSEa system, exploits Knowledge Cartography to inferring and storing inferred information. KIE allows for processing a terminology (TBox) and assertions (ABox). Information is stored in a relational database (PostgreSQL 8.0 has been exploited in the most up-to-date version of KIE).

In the following we present scenarios that take place in situations of database update (Tell) and query (Ask) in the context of the previously described database schema. KIE is able to handle queries available in the DIGUT language [8], a special language for querying the KASEA system, based on DIG/1.1 [9].

Tell queries that can be handled by the KASEA system are concept assertion of the form $C(a)$ and role assertions of the form $R(a, b)$.

In processing of a concept assertion $C(a), C$ has to be an expression built of constructs used in the terminology. Constructors of the form $\exists R . A$ are allowed only if a signature for this constructor has been determined. The course of actions is as follows:

1. Calculate a signature $s(C)$.

2. If $a$ is not in the database add it and assign it a signature $s(C)$. End the procedure.

3. Otherwise combine the signature of $C_{a}$ with $s(C)$ using logical AND operation.

4. If a signature of $C_{a}$ has been changed update the neighbourhood of $a$ (see below).

In processing of a concept assertion role assertions of the form $R(a, b)$ only neighbourhood update is performed.

Necessity of updating neighbourhood is a consequence of the fact that changing our knowledge about membership of the individual $a$ may change our knowledge about the individuals related to $a$. In the current version of the KASEA system a simple mechanism of positive and negative role checking has been applied. In positive role checking every pair $(a, b)$ related with $R$ is checked against all defined concepts of the form $\exists R$.C. If $b$ is a member of the concept $C$ the signature of $C_{a}$ is combined with $s(\exists R . C)$ using logical AND operation. In negative role checking every pair $(a, b)$ related with $R$ is checked against all defined concepts of the form $\exists R$.C. If $a$ is a member of the concept $\neg \exists R$.C the signature of $C_{b}$ is combined with a signature of $s(\neg C)$ using logical AND operation. 
The Knowledge Cartography - A new approach to reasoning over Description Logics ontologies 9

This process is recursively repeated if a signature of any individual has been changed. The process has to end in a finite number of steps because of the fact that the number of individuals is finite and each update may only decrease a number of "1"s in signatures of individuals being processed.

Almost all Ask operations can be brought down to subsumption checking. For example instance retrieval problem for concept $C$ can be viewed as finding all individuals $a$ such that $C_{a}$ is subsumed by $C$. In the course of subsumption checking section counters are exploited to do the preliminary selection of "candidate" signatures. Then bitwise Boolean operations are performed in order to check whether two signatures are in $\leq$ relation.

\section{Performance results}

The space and time complexity of processing a terminology by Cartographer is in the worst case exponential. Indeed, the maximum number of regions that the space can be divided into by $k$ concepts is $2^{k}$, which results in signatures of such a length. The corresponding terminology would consist of $k$ concepts that are not related to each other at all. However, such a case is very rare in practical terminologies (if found at all). Specifically, this would mean that any subset of concepts may have common instances, because no pair of concepts is declared disjoint. For instance, consider the terminology $\mathcal{T}$ containing one root concept and three direct subconcepts (axioms 1, 2, and 3 below):
1. Bird $\sqsubseteq$ Animal
4. Bird $\sqcap$ Fish $\equiv \perp$
2. Fish $\sqsubseteq$ Animal
5. Bird $\sqcap$ Mammal $\equiv \perp$
3. Mammal $\sqsubseteq$ Animal
6. Fish $\sqcap$ Mammal $\equiv \perp$

In this terminology, Bird, Fish and Mammal are unrelated, which means that we could declare one individual to be simultaneously a bird and a fish. Such a terminology would create a domain space with 9 regions (Fig. 3a). Let us add three disjoints to $\mathcal{T}$ (axioms 4, 5, and 6 above). The number of regions in the domain space decreased to 5 (Fig. 4 b). Actually, $\mathcal{T}$ is now a pure tree taxonomy, which reflects reality among animals.

a)

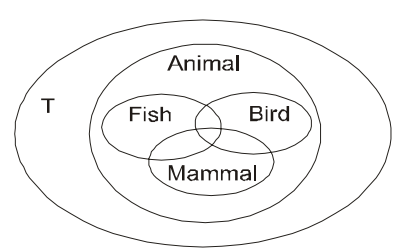

b)

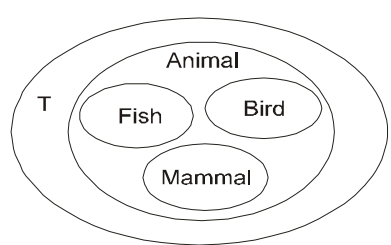


10 Krzysztof Goczyła, Teresa Grabowska, Wojciech Waloszek, Michal Zawadzki

Fig. 3. The division of a domain space for the exemplary terminology: a) without disjoints, and b) with disjoints

For taxonomies the signature size grows linearly with $k$. Our experiments show that the observed time complexity of map generation algorithm is proportional to $k \log k$ for taxonomies except of inherently quadratic $\left(\mathrm{O}\left(k^{2}\right)\right)$ signature generation phase (the algorithm has to generate $k$ signatures of length proportional to $k$ ). The results of terminology processing time are presented in Table 1 (all tests were performed on a PC with Celeron $2.4 \mathrm{GHz}$ and $256 \mathrm{MB}$ RAM). It is worth to notice that the time was independent on the order of axioms in terminologies, as special heuristics has been used to estimate the best possible ordering.

The ABox queries answering performance of Knowledge Inference Engine has been tested and compared with several freely available tools: Jena 2 [10], FaCT [11], [12], Racer [13]. The results of the tests are presented in Table 2 (FaCT is not included in the table because of its lack of support for ABoxes).

\begin{tabular}{|c|c|c|}
\hline $\begin{array}{c}\text { Number of concepts in } \\
\text { taxonomy }\end{array}$ & $\begin{array}{c}\text { Processing and post- } \\
\text { processing time [s] }\end{array}$ & $\begin{array}{c}\text { Signature generation } \\
\text { time [s] }\end{array}$ \\
\hline 3357 & 72 & $<1$ \\
\hline 82706 & 380 & 12 \\
\hline 184086 & 857 & 115 \\
\hline 545450 & 1386 & 2253 \\
\hline
\end{tabular}

Table 1. Results of efficiency experiments concerning signature generation for pure taxonomies.

\begin{tabular}{|l|c|c|c|c|c|c|}
\hline & \multicolumn{3}{|c|}{ Loading time [s] } & \multicolumn{3}{c|}{ Query processing time } \\
{$[\mathrm{s}]$}
\end{tabular}

Table 2. Results of efficiency experiments. Hyphens denote that the activity could not be completed within 2 hours.

The main difference between analysed reasoning algorithms is related to time of loading an ontology (ABox tell queries). The time of loading ontology is longer for KIE. In return we obtain a very short time of response. While Racer was unable to answer a query when 1000 individuals have been loaded, KIE in KASEA was able to process the same query for 11000 individuals in 1.4 seconds. 
The Knowledge Cartography - A new approach to reasoning over Description Logics ontologies 11

\section{Further Development}

Our present work concentrates on overcoming the limitations of the KASEA system. The most notable ones are: restriction of use of $\exists R$.C concepts in queries and the shortcomings of neighbourhood update mechanism.

Although the former limitation may seem inherent for Cartographic representation we work on overcoming it by using signatures with variable length. The range of $s$ function is in this way theoretically infinite, but longer signatures are assigned to complex concepts that are very unlikely to appear in client queries.

The latter limitation comes out from the fact that neighbourhood update mechanism is not fully OWA (Open World Assumption) compliant. Consider the ontology in Figure 5. It can be easily noticed that there are only two possible cases of membership of $a$ and $b$ individuals. Namely, $a$ can be a Man and $b$ a Women or alternatively $a$ can be a Woman and $b$ a Man. In both the cases $c$ is a member of Bisexual concept. That fact will not be inferred by the KASEA system because in the processing of Tell queries only signatures of direct neighbours are taken into consideration.

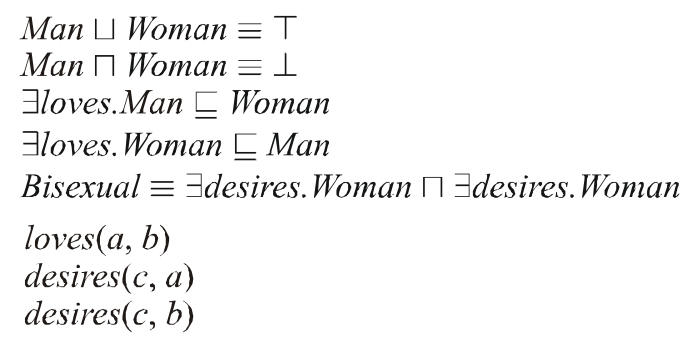

Fig. 4. An exemplary ontology showing limitations in OWA support of the KaSEA system

Our present work concentrates on overcoming the above limitations. Moreover we are gradually extending expressiveness of the KASEA system constructs in order to support such constructs as cardinality constraints, symmetric, transitive and functional roles so that KIE could be able to reason at least over the whole $\mathcal{S H \mathcal { I }}$ (a DL dialect that OWL is based on). We are also working on extending signature analysis on roles. Another research focuses on knowledge distribution and embedding trust issues into the knowledge base. 
12 Krzysztof Goczyla, Teresa Grabowska, Wojciech Waloszek, Michal Zawadzki

\section{References}

1. Baader F. A., McGuiness D. L., Nardi D., Patel-Schneider P. F.: The Description Logic Handbook: Theory, implementation, and applications, Cambridge University Press, 2003.

2. OWL - Web Ontology Language Guide, W3C, http://www/w3.org/2004/OWL

3. A Semantic Web Framework for Java, http://jena.sourceforge.net/

4. Goczyła K., Grabowska T., Waloszek W., Zawadzki M.: Issues related to Knowledge Management in e-health System, (in Polish). In: Software Engineering - New Challenges", Eds. J. Górski, A.. Wardziński, WNT 2004, Chap. XXVI, pp. 358-371.

5. S. Bechhofer, I. Horrocks, D. Turi Implementing the Instance Store, available at CEUR http://sunsite.informatik.rwth-aachen.de/Publications/CEUR-WS/Vol-115/

6. Goczyła K., Grabowska T., Waloszek W., Zawadzki M. The Cartographer Algorithm for Processing and Querying Description Logics Ontologies. Lecture Notes in Artificial Intelligence, Vol. June 2005, pp.

7. Bryant, R. E. Graph-based algorithms for boolean function manipulation, IEEE Transaction on Computers, 1986.

8. DIGUT Interface, Version 1.3. KMG@GUT Technical Report, 2005. Available at $\mathrm{http} / / / \mathrm{km}$.pg.gda.pl/km/DIGUT_Interface_1.3.pdf

9. Bechhofer S.: The DIG Description Logic Interface: DIG/1.1, University of Manchester, February 7, 2003.

10. D. Reynolds: Jena 2 Inference support, 2004, also available at WWW site http://jena.sourceforge.net/inference/index.html

11. I. Horrocks, P. F. Patel-Schneider: Optimising Propositional Modal Satisfiability for Description Logic Subsumption, 1998, file available at the following Internet site: http://www.cs.man.ac.uk/ horrocks/Publications/download/1998/aisc98.ps.gz

12. I. Horrocks: FaCT Reference Manual v1.6, August 1998, Included in FaCT archive from http://www.cs.man.ac.uk/ horrocks/FaCT/

13. V. Haarslev, R. Möller: RACER User's Guide and Reference Manual, September 17, 2003, http://www.cs.concordia.ca/ haarslev/racer/racer-manual-1-7-7.pdf 